\title{
Perfil e trajetória dos egressos de programas de residência das áreas básicas: um corte transversal
}

\section{Profile and professional career of graduates from a residency program of the basic medical areas: a cross section study}

Eduardo Jorge da Fonseca Lima1,2 (D) | eduardojorge@imip.org.br Pedro Jorge Serra da Fonseca Lima' (1) pedrojorgesflima@gmail.com

Pedro Henrique Alves de Andrade ${ }^{1}$ (D) pedroalves_andrade@hotmail.com Lucas Miranda Castro' (1) Imc19051998@hotmail.com Afra Suassuna Fernandes² (D) afra.suassuna@imip.org.br

\section{RESUMO}

Introdução: A residência médica é reconhecida como o "padrão ouro" para a formação de especialistas. O estudo do perfil dos egressos da residência médica é importante para identificar potencialidades e fragilidades da especialização.

Objetivo: Este estudo teve como objetivo conhecer o perfil e a satisfação profissional dos egressos dos programas de residência das áreas básicas de um hospital-escola do Nordeste.

Método: Trata-se de estudo de corte transversal que utilizou a plataforma eletrônica Survey. Incluíram-se residentes das áreas básicas que concluíram o programa no período de 2013 a 2017. OTermo de Consentimento Livre e Esclarecido foi enviado com o formulário. Realizaram-se análises descritivas das variáveis, e os dados foram apresentados em frequências absoluta e relativa. O trabalho foi aprovado pelo Comitê de Ética do IMIP.

Resultados: Dentre os 194 egressos, tivemos a adesão de 79 (40,72\%). Com relação aos participantes do estudo: 73,4\% eram do gênero feminino e $60,8 \%$ já estavam casados. Destacamos que 55,7\% informaram que tinham uma renda mensal de dez a 20 salários mínimos. Dos egressos, 54 $(68,4 \%)$ tinham cursado graduação em instituição de ensino superior privada. Sobre a pós-graduação stricto sensu, 19 egressos (21,7\%) tinham mestrado. Sobre a atuação profissional, $93,7 \%$ exercem a especialidade e $54(68,4 \%)$ trabalham no estado onde cursaram o programa. Em relação ao serviço público, 64,6\% são vinculados ao Sistema Único de Saúde do estado de Pernambuco. Sobre a quantidade de horas de trabalho semanais, $43 \%$ trabalham entre 40 e 60 horas. Cerca de $75 \%$ dos egressos afirmaram que cursariam o programa novamente na instituição e declararam que a realização da residência facilitou a vida profissional deles.

Conclusão: A monitoração periódica de egressos de programa de residência é um instrumento útil para avaliação do programa e permite monitoramento das intervenções implementadas, viabilizando inclusive a obtenção de informações que ajudem no planejamento de novos programas.

Palavras-chave: Educação; Recursos Humanos em Saúde; Residência Médica.

\section{ABSTRACT}

Introduction: medical residency is known as the "gold standard" for the qualification of specialists. Research into the profile of medical residency program graduates is important to identify potentialities and fragilities of the specialization.

Objective: The objective of this study was to understand the profile and professional satisfaction of the graduates from the residency programs of basic medical areas of a teaching hospital in the northeast of Brazil.

Methods: a cross-sectional study was conducted through an online questionnaire that was sent to the participants via electronic mail. The study population consisted of residency program graduates of the basic medical areas who graduated between 2013 and 2017. The Informed Consent Form was sent together with the form. Descriptive analyses were performed and data were presented as relative and absolute frequencies. The study was approved by the Research Ethics Committee of the IMIP.

Results: of the 194 graduates, 79 (40.72\%) answered the questionnaire. $73.4 \%$ were female and $60.8 \%$ were married. Approximately 55.7\% of the graduates earned salaries corresponding to 11 and 22 minimum monthly wages. 54 graduates (68.4\%) had attended a private medical school. Regarding stricto sensu postgraduate studies, 19 (21.7\%) had a master's degree. 93.7\% are practicing their medical specialization and 54 (68.4\%) still live in Pernambuco. Regarding public service, $64.6 \%$ are linked to the Unified Health System of Pernambuco state. $43 \%$ of the graduates worked between 40 and $60 \mathrm{~h}$ per week. About $75 \%$ of the graduates stated that they would attend the program again at the hospital and declared that completing the residency at the institution facilitated their professional life.

Conclusion: the periodic monitoring of graduates from a residency program is a useful tool for evaluating the program and allows for surveillance of implemented interventions

Keywords: Education; Human Resources in Health; Medical Residency.

${ }^{1}$ Faculdade Pernambucana de Saúde, Recife, Pernambuco, Brasil.

2 Instituto de Medicina Integral Professor Fernando Figueira, Recife, Pernambuco, Brasil.

Editora-chefe: Rosiane Viana Zuza Diniz ～～～Editor associado: Roberto Zonato Esteves

Recebido em 16/10/20; Aceito em 08/01/21. | Avaliado pelo processo de double blind review. 


\section{INTRODUÇÃO}

A residência médica $(\mathrm{RM})$ é reconhecida como uma modalidade de pós-graduação e considerada o "padrão ouro" para a formação de especialistas. Seu histórico remonta ao ano de 1889, no John's Hopkins Hospital, onde surgiram os primeiros programas de residência coordenados por Halsted e Osler nas áreas de cirurgia e clínica médica, respectivamente1. Em 1945, foi implantado o primeiro Programa de Residência Médica (PRM) no Brasil: em ortopedia, no Hospital das Clínicas da Universidade de São Paulo. A seguir, no Instituto de Previdência e Assistência ao Servidor do Estado do Rio de Janeiro, em 1948, criaram-se os PRMs em cirurgia geral, clínica médica, pediatria e obstetrícia/ginecologia'.

Desde 1977, todos os PRMs no Brasil estão subordinados à Comissão Nacional de Residência Médica (CNRM), que estabeleceu normas e critérios para o credenciamento de programas. Nos termos do Decreto no 80.281/77, a RM constitui um curso de pós-graduação destinado a médicos e caracterizado por treinamento em serviço do médico residente, cujas atividades são sempre supervisionadas por profissionais de reconhecida competência ética e técnica'. Os médicos especialistas devem estar preparados para atender às demandas de saúde da população, e, portanto, as competências adquiridas no PRM são imprescindíveis para sua prática clínica².

O estudo do perfil dos egressos da RM é importante para identificar potencialidades e fragilidades da especialização, podendo extrapolar para a residência as recomendações do Sistema Nacional de Avaliação da Educação Superior (Sinaes) voltadas à graduação ${ }^{3}$.

O Instituto de Medicina Integral Professor Fernando Figueira (IMIP) foi fundado em 1960 e é uma entidade filantrópica, que atua nas áreas de assistência médicosocial, ensino, pesquisa e extensão comunitária. Voltado para o atendimento da população carente pernambucana, o Complexo Hospitalar do IMIP é reconhecido como uma das estruturas hospitalares mais importantes do país, sendo centro de referência assistencial em diversas especialidades médicas ${ }^{4}$.

O PRM em pediatria do IMIP foi iniciado em 1966, tendo ao longo desse período se destacado como um dos programas de referência na especialidade no país. Atualmente, o IMIP dispõe de 48 PRMs. Do ponto de vista assistencial, a instituição é voltada para o atendimento da população carente pernambucana ao prestar assistência integral à saúde da criança, da mulher e do adulto.

Desde a sua implantação, o modelo educacional da RM obedeceu a uma programação básica, com três elementos importantes: a população a ser assistida, a estrutura hospitalar adequada para o ensino e preceptores qualificados ${ }^{5}$. Os médicos especialistas devem estar preparados para atender às demandas de saúde da população, e, portanto, a formação obtida durante a realização dos PRMs é imprescindível para sua prática clínica ${ }^{6-9}$.

Apesar da existência de inúmeros serviços com RM no Brasil, praticamente não há programas regulares de acompanhamento de avaliação da qualidade, exceto as visitas de credenciamento do Ministério da Educação ${ }^{8,9}$. Para conhecer a qualidade de um programa de formação profissional, é fundamental conhecer o perfil de egresso profissional treinado nos programas e a trajetória futura desse especialista.

A American Medical Association objetivou conhecer o grau de dificuldade que os médicos egressos de PRMs encontravam para se inserir nesse mercado e verificou que $67 \%$ atuavam na especialidade correspondente ao seu último PRM, 15,5\% optaram pela carreira acadêmica, 5\% atuavam em especialidade diferente da formação na RM, 5,1\% declararam ter outros planos profissionais e 7,1\% não conseguiram ingressar no mercado de trabalho. Do total de médicos que conseguiram emprego na sua área de formação, 22,4\% relataram ter tido significante dificuldade para ingressar no mercado 5 .

Um estudo semelhante realizado no Departamento de Pediatria da Faculdade de Medicina da Universidade de São Paulo ${ }^{10}$ com egressos da residência constatou que $64 \%$ dos especializados continuavam atuando na cidade onde foi realizado o programa, $78 \%$ se consideravam profissionalmente realizados e $22 \%$ se sentiam parcialmente realizados em virtude da renda mensal inferior à esperada e por não terem iniciado/concluído outra pós-graduação. Destacamos que $54 \%$ dos egressos da residência exerciam função docente, principalmente em serviços públicos ${ }^{10}$.

Outro aspecto relevante nos estudos sobre egressos refere-se aos dados de saúde mental ${ }^{11}$. Destaca-se o fato de as profissões de saúde serem desgastantes, com grande dedicação de tempo, envolvimento e muita responsabilidade pessoal, além do contato constante com o sofrimento de pacientes e familiares. Uma pesquisa realizada pelo Conselho Federal de Medicina ${ }^{12}$ mostrou que a grande maioria dos médicos considera fundamental uma boa combinação entre trabalho e vida pessoal. Para $83,6 \%$ deles, a "capacidade de obter um equilíbrio entre a profissão e a vida pessoal" é um dos fatores mais importantes para um "exercício profissional satisfatório e gratificante". O segundo fator mais citado - assinalado por $64,2 \%$ dos egressos - foi "ter uma jornada de trabalho flexível", enquanto 49,4\% citaram "ter competência técnica". A possibilidade de "exercer o ensino" foi assinalada por $43,6 \%$, enquanto $42,8 \%$ dos novos médicos mencionaram "contar com um sistema de saúde que dê assistência adequada para meus pacientes"12.

Portanto, o acompanhamento regular e sistemático de egressos é uma prática ainda incomum em diversas áreas, especialmente entre instituições formadoras de médicos 
residentes. Existem poucos dados e publicações com informações sobre o perfil dos egressos de PRMs e seu desempenho ao longo da carreira, apesar da grande importância do tema ${ }^{6-9}$.

Os objetivos do nosso estudo foram conhecer o perfil e a satisfação profissional dos egressos dos PRMs das áreas básicas do IMIP, contribuindo para análise da inserção desse profissional no Sistema Único de Saúde (SUS), e verificar o grau de satisfação com o programa cursado e com sua qualidade de vida atual.

\section{MÉTODOS}

Realizou-se um estudo de corte transversal no qual se utilizou a plataforma eletrônica Survey para a coleta dos dados. A população de estudo foi composta por todos os residentes das quatro áreas básicas (clínica médica, pediatria, ginecologia/obstetrícia e cirurgia geral) que concluíram os PRMs no IMIP, no período de 2013 a 2017. Portanto, os critérios de inclusão foram todos os residentes que concluíram esses programas no período citado, sendo excluídos apenas os que não concordaram em responder ao questionário. De um total de 194 concluintes (69 pediatras, 67 ginecologistas e obstetras, 39 médicos clínicos e 19 cirurgiões gerais), tivemos a adesão de 79 egressos. Não realizamos análise inferencial porque a amostra dos egressos não permitiu comparações entre as áreas cursadas ou outro tipo de análise.

Um questionário eletrônico foi enviado para o e-mail pessoal dos participantes por meio da plataforma disponibilizada pela Faculdade Pernambucana de Saúde (FPS), e a coleta de dados ocorreu durante os meses de novembro de 2018 a fevereiro de 2019. Reenviaram-se os e-mails semanalmente aos que não responderam e efetuaram-se ligações por telefone para os residentes que tinham iniciado a pesquisa, mas não a concluíram, solicitando a adesão ao estudo e o término das respostas.

O questionário foi composto por perguntas estruturadas e abertas com respostas curtas sobre as características sociodemográficas, acadêmicas e profissionais, a satisfação com a profissão e a saúde do egresso. O questionário foi previamente aplicado a médicos residentes do hospital-escola da instituição em um projeto-piloto composto por 20 participantes, com o objetivo de avaliar a clareza e a pertinência das perguntas antes da elaboração da versão definitiva. O Termo de Consentimento Livre e Esclarecido (TCLE) foi enviado com o e-mail, sendo necessária a leitura e concordância com os termos do estudo para ter acesso ao questionário na plataforma utilizada.

Os dados coletados foram armazenados na própria plataforma disponibilizada e posteriormente organizados em planilha do software Microsoft Office Excel. O programa utilizado para análise estatística foi o Epilnfo versão 7.2.2.2. Realizaram-se análises descritivas das variáveis do estudo, e apresentou-se a sua distribuição de frequência.

O presente trabalho foi aprovado pelo Comitê de Ética e Pesquisa em Seres Humanos do IMIP, sendo aprovado pelo Parecer $n^{\circ} 2.710 .534$.

\section{RESULTADOS}

Dos 194 egressos que concluíram a residência no período de 2013 a 2017 no IMIP, 79 (40,72\%) aderiram ao questionário enviado com preenchimento completo.

No Gráfico 1, descrevemos a distribuição por programa de residência da nossa amostra, em que o maior percentual de respostas foi dos egressos do PRM em pediatria (41\%), que é o programa com maior número de residentes da instituição.

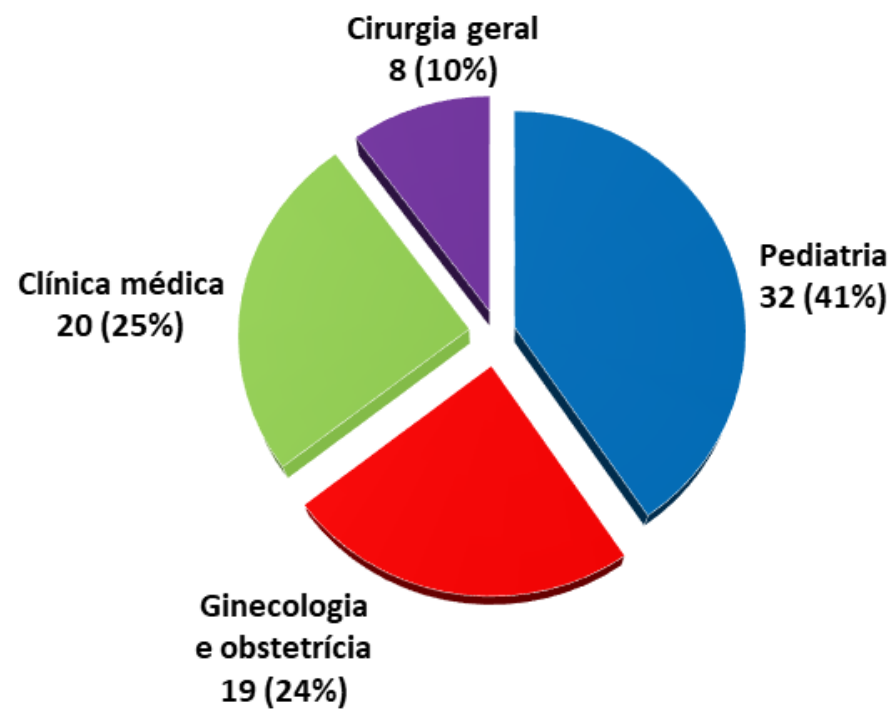

Gráfico 1. Distribuição por programa dos egressos de residência médica de quatro áreas básicas do Instituto de Medicina Integral Professor Fernando Figueira (IMIP) no período de 2013 a 2017, Recife, 2019 
A análise do perfil sociodemográfico dos egressos da instituição está descrita na Tabela 1. Salientamos que $73,4 \%$ eram do gênero feminino, mais de $60,8 \%$ eram casados, embora $64,6 \%$ ainda não tivessem filhos. Destacamos que $55,7 \%$ dos egressos informaram que sua renda mensal se situava entre dez e 20 salários mínimos por mês.

O histórico da formação acadêmica desde a graduação até a realização de pós-graduação stricto sensu está demonstrado na Tabela 2. Dos egressos que responderam ao nosso inquérito, 54 (68,4\%) tinham cursado a graduação em uma instituição de ensino superior (IES) privada, 86,1\% foram aprovados no processo seletivo para a RM no mesmo ano em que concluíram a graduação e apenas cinco $(6,3 \%)$ utilizaram - Programa de Valorização dos Profissionais da Atenção Básica (Provab) para essa aprovação. Em relação a ter cursado pós-graduação stricto sensu, 19 egressos (21,7\%) já tinham realizado mestrado.

A inserção no mercado de trabalho está detalhada na Tabela 3. Ressaltamos que $93,7 \%$ dos egressos estão exercendo a especialidade da área básica cursada, dos quais $54(68,4 \%)$ trabalham em Pernambuco. Em relação ao serviço público, $64,6 \%$ são vinculados a esse regime de trabalho, sendo a maioria servidora da Secretaria Estadual de Saúde de Pernambuco (SES-PE). Sobre a permanência no IMIP ou na rede IMIP hospitalar, verificou-se que $44,3 \%$ exerciam suas atividades nessas unidades.

A Tabela 4 apresenta a percepção dos participantes sobre o programa cursado, a carga horária e a satisfação pessoal. Dos egressos, $86,1 \%$ acreditam que ter realizado programa de residência no IMIP facilitou a vida profissional deles. Em relação à renda atual, 77,2\% consideram satisfatória ou muito satisfatória, e todos responderam que consideram o exercício da profissão gratificante.

Analisaram-se as principais vantagens e desvantagens de ter realizado residência no IMIP. Os fatores citados com maior frequência como vantagem foram: número de pacientes, diversidade de casos, qualidade da preceptoria, infraestrutura do hospital e qualidade das atividades teóricas. Em relação às desvantagens, mencionaram-se: carga horária desgastante, excesso de pacientes e dificuldades na infraestrutura.

Também verificamos aspectos de hábitos da vida atual do egresso. Em relação à quantidade de horas de sono por dia, destacamos que 44 (55,7\%) dormiam entre seis e oito horas diárias. Ao serem questionados se a qualidade de vida melhorou após o término da residência, 52 (65,8\%) responderam afirmativamente. A prática de exercício físico regular foi evidenciada em apenas 39 $(49,4 \%)$ dos egressos. Ressaltamos ainda que apenas 32 egressos $(40,5 \%)$ relataram que tempo livre disponível é suficiente para a saúde física e mental.
Tabela 1. Características sociodemográficas dos egressos dos programas de residência médica de quatro áreas básicas do Instituto de Medicina Integral Professor Fernando Figueira (IMIP) no período de 2013 a 2017, Recife, 2019

\begin{tabular}{lccc}
\hline & Biológicas & $\begin{array}{c}\mathbf{N} \\
(\mathbf{n}=79)\end{array}$ & $\%$ \\
\hline Idade & & \\
\hline & $26 \mid-29$ & 20 & 25,3 \\
\hline $29 \mid-32$ & 37 & 46,8 \\
\hline $32 \mid-35$ & 20 & 25,3 \\
\hline Gênero & Acima de 35 anos & 2 & 2,5 \\
\hline \multicolumn{3}{c}{ Masculino } \\
\hline Feminino & 21 \\
\hline Sociodemográficas & 26,6 \\
\hline
\end{tabular}

\section{Estado civil}

\begin{tabular}{ccc}
\hline Solteiro & 27 & 34,2 \\
\hline Casado & 48 & 60,8 \\
\hline União estável & 3 & 3,8 \\
\hline Divorciado & 0 & 0,0 \\
\hline Sem resposta & 1 & 1,3 \\
\hline Número de filhos & &
\end{tabular}

Número de filhos

\begin{tabular}{ccc}
\hline Não tenho filhos & 51 & 64,6 \\
\hline 1 filho & 22 & 27,8 \\
\hline 2 filhos & 6 & 7,6 \\
\hline 3 filhos & 0 & 0,0 \\
\hline Mais do que 3 filhos & 0 & 0,0 \\
\hline Renda mensal (R\$) & & \\
\hline 5.000 |- 10.000 & 22 & 27,8 \\
\hline $10.000 \mid-20.000$ & 44 & 55,7 \\
\hline $20.000 \mid-30.000$ & 11 & 13,9 \\
\hline acima de 30.000 & 2 & 2,5 \\
\hline
\end{tabular}

Residência no início do programa

\begin{tabular}{ccc}
\hline Recife/Grande Recife & 36 & 45,6 \\
\hline Interior de Pernambuco & 2 & 2,5 \\
\hline Outro estado do Brasil & 12 & 15,2 \\
\hline Outro país & 0 & 0,0 \\
\hline Sem resposta & 29 & 36,7 \\
\hline
\end{tabular}

Residência atual

\begin{tabular}{ccc}
\hline Recife/Grande Recife & 51 & 64,6 \\
\hline Interior de Pernambuco & 0 & 0,0 \\
\hline Outro estado do Brasil & 25 & 31,6 \\
\hline Outro país & 1 & 1,3 \\
\hline Sem resposta & 2 & 2,5 \\
\hline
\end{tabular}


Tabela 2. Formação acadêmica dos egressos dos programas de residência médica de quatro áreas básicas do Instituto de Medicina Integral Professor Fernando Figueira (IMIP) no período de 2013 a 2017, Recife, 2019

\begin{tabular}{|c|c|c|}
\hline Graduação & $\begin{array}{c}N \\
(n=79)\end{array}$ & $\%$ \\
\hline \multicolumn{3}{|l|}{ Tipo de IES } \\
\hline Pública & 25 & 31,6 \\
\hline Privada & 54 & 68,4 \\
\hline \multicolumn{3}{|l|}{ Instituição } \\
\hline $\begin{array}{l}\text { Universidade Federal de Pernambuco } \\
\text { (UFPE) }\end{array}$ & 13 & 16,5 \\
\hline Universidade de Pernambuco (UPE) & 17 & 21,5 \\
\hline FPS & 20 & 25,3 \\
\hline $\begin{array}{l}\text { Universidade Federal do Vale do São } \\
\text { Francisco (Univasf) }\end{array}$ & 03 & 3,8 \\
\hline Outros estados & 26 & 32,9 \\
\hline \multicolumn{3}{|l|}{ Residência } \\
\hline \multicolumn{3}{|l|}{ Ano do processo seletivo } \\
\hline No mesmo ano em que concluiu a graduação & 68 & 86,1 \\
\hline Um ano após o término da graduação & 07 & 8,9 \\
\hline Dois anos após o término da graduação & 04 & 5,1 \\
\hline Três anos após o término da graduação & 0 & 0,0 \\
\hline $\begin{array}{l}\text { Quatro ou mais anos após o término da } \\
\text { graduação }\end{array}$ & 0 & 0,0 \\
\hline
\end{tabular}

Utilização de pontos obtidos por meio do Provab

\begin{tabular}{ccc} 
Sim & 5 & 6,3 \\
\hline Não & 74 & 93,7 \\
\hline
\end{tabular}

Ano de conclusão da residência no IMIP

\begin{tabular}{rrr}
\hline 2013 & 13 & 16,5 \\
\hline 2014 & 16 & 20,3 \\
\hline 2015 & 17 & 21,5 \\
\hline 2016 & 14 & 17,7 \\
\hline 2017 & 19 & 24,1 \\
\hline
\end{tabular}

Produção científica durante o PRM

\begin{tabular}{ccc}
\hline Sim & 44 & 55,7 \\
\hline Não & 35 & 44,3 \\
\hline
\end{tabular}

Tipos de produção científica durante o PRM

\begin{tabular}{ccc}
\hline Artigo em revista indexada & 10 & 16,4 \\
\hline Tema oral em congresso da especialidade & 13 & 21,3 \\
\hline Pôster em um congresso da especialidade & 38 & 62,3 \\
\hline Realização de segundo PRM & & \\
\hline Sim & 60 & 75,9 \\
\hline Não & 19 & 24,1 \\
\hline Título de especialista pela sociedade da área & \\
\hline Sim & 38 & 48,1 \\
\hline Não & 41 & 51,9 \\
\hline
\end{tabular}

Continua...
Tabela 2. (Continuação) Formação acadêmica dos egressos dos programas de residência médica de quatro áreas básicas do Instituto de Medicina Integral Professor Fernando Figueira (IMIP) no período de 2013 a 2017, Recife, 2019

\begin{tabular}{ccc}
\hline Residência & $\begin{array}{c}\mathrm{N} \\
(\mathrm{n}=79)\end{array}$ & $\%$ \\
\hline $\begin{array}{l}\text { Registro da especialidade no Conselho Regional de } \\
\text { Medicina (CRM) }\end{array}$ & \\
\hline Sim & 35 & 44,3 \\
\hline Não & 44 & 55,7 \\
\hline Pós-graduação stricto sensu & \\
\hline Mestrado & \\
\hline Sim & 19 \\
\hline Não & 21,7 \\
\hline Mestrado associado à residência do IMIP $(\boldsymbol{n}=19)$ & 78,3 \\
\hline Sim & 05 & 26,3 \\
\hline Não & 14 & 73,7 \\
\hline Doutorado & \\
\hline Sim & 03 \\
\hline Não & 36,8 \\
\hline
\end{tabular}

Tabela 3. Inserção no mercado de trabalho dos egressos dos programas de residência médica de quatro áreas básicas do Instituto de Medicina Integral Professor Fernando Figueira (IMIP) no período de 2013 a 2017, Recife, 2019

\begin{tabular}{|c|c|c|}
\hline Atuação & $\begin{array}{c}N \\
(n=79)\end{array}$ & $\%$ \\
\hline \multicolumn{3}{|l|}{ Exerce a especialidade obtida no PRM-IMIP } \\
\hline Sim & 74 & 93,7 \\
\hline Não & 05 & 6,3 \\
\hline \multicolumn{3}{|l|}{ Local onde exerce a profissão } \\
\hline Pernambuco & 54 & 68,4 \\
\hline Outro estado do Brasil & 24 & 30,4 \\
\hline Outro país & 0 & 0,0 \\
\hline Não exerce a medicina & 0 & 0,0 \\
\hline Sem resposta & 01 & 1,3 \\
\hline \multicolumn{3}{|l|}{ Sobre os que atuam em PE $(n=54)$} \\
\hline Apenas em Recife & 37 & 68,5 \\
\hline Recife e região metropolitana & 12 & 22,2 \\
\hline Recife e interior fora da região metropolitana & 05 & 9,3 \\
\hline Apenas no interior do estado & 0 & 0,0 \\
\hline \multicolumn{3}{|c|}{ Quanto à(s) instituição(ões) onde exerce a profissão } \\
\hline Apenas da rede pública & 22 & 27,8 \\
\hline Apenas da rede privada & 02 & 2,5 \\
\hline Em ambas as redes & 54 & 68,4 \\
\hline Não se aplica & 01 & 1,3 \\
\hline
\end{tabular}


Tabela 3. (Continuação) Inserção no mercado de trabalho dos egressos dos programas de residência médica de quatro áreas básicas do Instituto de Medicina Integral Professor Fernando Figueira (IMIP) no período de 2013 a 2017, Recife, 2019

$$
\text { Atuação } \quad \begin{gathered}
\mathrm{N} \\
(\mathrm{n}=79)
\end{gathered} \quad \%
$$

Servidor público

\begin{tabular}{lll} 
Sim & 51 & 64,6 \\
Não & 28 & 35,4 \\
\hline
\end{tabular}

Servidor público vinculado a: $(n=51)$

\begin{tabular}{ccc}
\hline SES Pernambuco & 25 & 49,0 \\
\hline Recife & 03 & 5,9 \\
\hline Outra instituição & 05 & 9,8 \\
\hline Outros & 18 & 35,3 \\
\hline Médico do IMIP ou gestão hospitalar IMIP & & \\
\hline Sim & 35 & 44,3 \\
\hline Não & 44 & 55,7 \\
\hline
\end{tabular}

\section{Número de vínculos empregatícios}

\begin{tabular}{ccc}
\hline Somente um & 18 & 22,8 \\
\hline Dois & 25 & 31,6 \\
\hline Mais de dois & 36 & 45,6 \\
\hline
\end{tabular}

\section{Horas de trabalho semanais}

\begin{tabular}{ccc}
\hline Até 24 & 02 & 2,5 \\
\hline $24 \mid-32$ & 02 & 2,5 \\
\hline $32 \mid-40$ & 10 & 12,7 \\
\hline $40 \mid-60$ & 34 & 43,0 \\
\hline $60 \mid-80$ & 28 & 35,4 \\
\hline Não se aplica & 03 & 3,8 \\
\hline Sim & & \\
\hline Não & 71 & 89,9 \\
\hline
\end{tabular}

Horas correspondentes a plantões $(n=71)$

\begin{tabular}{ccc}
\hline Até 12 & 14 & 19,7 \\
\hline $18 \mid-24$ & 15 & 21,1 \\
\hline $24 \mid-36$ & 14 & 19,7 \\
\hline $36 \mid-48$ & 13 & 18,3 \\
\hline Acima de 48 & 8 & 11,3 \\
\hline Sem resposta & 7 & 9,9 \\
\hline
\end{tabular}

Plantões noturnos $(n=71)$

\begin{tabular}{ccc}
\hline Sim & 60 & 84,5 \\
\hline Não & 11 & 15,5 \\
\hline
\end{tabular}

Plantões em finais de semana $(n=71)$

\begin{tabular}{lll} 
Sim & 54 & 76,1 \\
Não & 17 & 23,9 \\
\hline
\end{tabular}

Tabela 3. (Continuação) Inserção no mercado de trabalho dos egressos dos programas de residência médica de quatro áreas básicas do Instituto de Medicina Integral Professor Fernando Figueira (IMIP) no período de 2013 a 2017, Recife, 2019

Atividades de ensino

$$
\begin{aligned}
& \mathrm{N} \\
& (\mathrm{n}=79)
\end{aligned}
$$

Docência na graduação de Medicina

\begin{tabular}{ccc}
\hline Sim & 21 & 26,6 \\
\hline Não & 58 & 73,4 \\
\hline Preceptoria em hospital de ensino & & \\
\hline Sim & 43 & 54,4 \\
\hline Não & 36 & 45,6 \\
\hline
\end{tabular}

Tabela 4. Percepção sobrea residência cursada ea especialidade dos egressos dos programas de residência médica de quatro áreas básicas do Instituto de Medicina Integral Professor Fernando Figueira (IMIP) no período de 2013 a 2017, Recife, 2019

$$
\begin{gathered}
N \\
(n=79)
\end{gathered}
$$

\section{Cursaria o PRM na área básica novamente}

\begin{tabular}{ccc}
\hline Sim & 59 & 74,7 \\
\hline Não & 20 & 25,3 \\
\hline
\end{tabular}

Cursaria o PRM na área básica novamente no IMIP

\begin{tabular}{ccc}
\hline Sim & 58 & 73,4 \\
\hline Não & 2 & 2,5 \\
\hline Sem resposta & 19 & 24,1 \\
\hline
\end{tabular}

\section{Opinião sobre ter realizado o PRM no IMIP}

\begin{tabular}{ccc} 
Facilitou a minha vida profissional & 68 & 86,1 \\
\hline Dificultou a minha vida profissional & 1 & 1,3 \\
\hline $\begin{array}{c}\text { Nem facilitou nem dificultou a minha } \\
\text { vida profissional }\end{array}$ & 10 & 12,7 \\
\hline
\end{tabular}

Dificuldade de inserção no mercado de trabalho público

\begin{tabular}{ccc}
\hline Sim & 15 & 19,0 \\
\hline Não & 64 & 81,0 \\
\hline
\end{tabular}

Dificuldade de inserção no mercado de trabalho privado

\begin{tabular}{lll} 
Sim & 14 & 17,7 \\
Não & 65 & 82,3 \\
\hline
\end{tabular}

\section{Atualmente, sua carga horária de trabalho comparada ao} período da residência é:

\begin{tabular}{ccc}
\hline Menor & 44 & 55,7 \\
\hline Maior & 11 & 13,9 \\
\hline Igual & 24 & 30,4 \\
\hline & & Continua...
\end{tabular}


Tabela 4. (Continuação) Percepção sobre a residência cursada e a especialidade dos egressos dos programas de residência médica de quatro áreas básicas do Instituto de Medicina Integral Professor Fernando Figueira (IMIP) no período de 2013 a 2017, Recife, 2019

\begin{tabular}{|c|c|c|}
\hline & $\begin{array}{c}N \\
(n=79)\end{array}$ & $\%$ \\
\hline \multicolumn{3}{|l|}{ Satisfação com a renda pessoal } \\
\hline Muito satisfatória & 15 & 19,0 \\
\hline Satisfatória & 46 & 58,2 \\
\hline Regular & 11 & 13,9 \\
\hline Insatisfatória & 7 & 8,9 \\
\hline Muito insatisfatória & 0 & 0,0 \\
\hline \multicolumn{3}{|c|}{$\begin{array}{l}\text { Considera o exercício da profissão } \\
\text { gratificante? }\end{array}$} \\
\hline Sim & 79 & 100 \\
\hline Não & 0 & 0 \\
\hline \multicolumn{3}{|c|}{$\begin{array}{l}\text { O exercício da profissão correspondeu às } \\
\text { expectativas }\end{array}$} \\
\hline Sim & 67 & 84,8 \\
\hline Não & 12 & 15,2 \\
\hline
\end{tabular}

\section{DISCUSSÃO}

A RM, inserida no escopo de pós-graduação lato sensu, caracteriza-se por ser equivalente à especialização e permite a concessão do título de especialista. É um programa de treinamento com carga horária semanal de 60 horas e visa transformar o recém-egresso da escola médica, formado com as competências de um médico generalista, em um especialista de determinada área. O processo de ensino-aprendizado é baseado fundamentalmente na prática médica supervisionada. Dessa forma, conhecer o perfil do egresso dos PRMs contribui de forma importante para uma análise dessa formação ${ }^{13}$.

No nosso estudo, de um total de 194 especialistas concluintes no período estudado, 79 egressos (40,72\%) participaram da pesquisa, respondendo integralmente ao questionário enviado via correio eletrônico. Esse número foi superior àqueles encontrados em outras pesquisas que utilizaram o mesmo desenho de estudo, a exemplo da avaliação do perfil dos egressos feita por Koch et al. ${ }^{9}$ na Faculdade de Medicina da Universidade de São Paulo, que contou com a resposta de apenas 33,6\% dos entrevistados. Dos programas oferecidos pelo IMIP, a pediatria foi a área com o maior percentual de respostas (40,50\%), já que é a especialidade com maior número de vagas oferecidas anualmente pela instituição.

Com relação ao gênero dos participantes, a maior parte da amostra (73,41\%) foi composta por mulheres, resultado que está em consonância com o perfil do jovem profissional médico em todo o mundo, como é o caso do estudo realizado por Smith et al. ${ }^{14}$ com os egressos de ortopedia do programa Kaiser Permanente em Havard, no estado da Califórnia, nos Estados Unidos. A tendência mundial iniciada na década de 1980 e conhecida como "feminização da profissão médica"15,16 ilustra a evolução da mulher no mercado de trabalho e sua posição de protagonista na construção de uma nova visão da saúde.

A idade média dos egressos foi majoritariamente de 29 a 32 anos (46,83\%) com apenas dois apresentando idade superior a 35 anos, evidenciando que a formação de graduação e a primeira residência na área básica ocorrem ao redor dos 30 anos de idade. Essa característica também foi observada em outros estudos, como na análise do perfil dos egressos da RM em cirurgia geral da Universidade de Ribeirão Preto, cuja média de idade foi de 31,7 anos ${ }^{17}$.

Um aspecto relevante demonstrado no estudo foi a confirmação da tendência de que o PRM tem a capacidade de fixar o residente na região. Enquanto no início do programa 38 $(48,10 \%)$ residiam em Pernambuco, atualmente 51 dos egressos $(64,55 \%)$ moram no estado. Em estudo realizado no Acre e em Roraima, observou-se a taxa de fixação de praticamente $100 \%$ dos médicos que não eram naturais dos estados, mas que fizeram a RM em ginecologia e obstetrícia, medicina da família e comunidade ou pediatria nesses locais ${ }^{18}$.

Esse aspecto da fixação do profissional pela RM foi reconhecido em nosso país e fundamentou a necessidade de implementar o Programa Nacional de Apoio à Formação de Médicos Especialistas em Áreas Estratégicas (Pró-Residência), projeto dos Ministérios da Saúde e Educação com apoio do Conselho Nacional de Secretários de Saúde (Conass) e do Conselho Nacional de Secretarias Municipais de Saúde (Conasems), que oferecia bolsas de RM em áreas definidas como prioritárias para o SUS e carentes de determinados especialistas ${ }^{19}$.

Mais da metade dos egressos (55,69\%) tiveram alguma produção científica durante o PRM nas áreas básicas estudadas. Pesquisas que avaliam essa produção em programas de subespecialidade encontraram resultados melhores, como na avaliação dos PRMs em cirurgia plástica do Distrito Federal, em que identificaram $66,7 \%$ de residentes com apresentações em eventos científicos ${ }^{20}$. A produção científica durante a residência representa um papel importante na formação do médico especialista e na aplicação do conhecimento, constituindo também alicerces para a opção de continuidade da pós-graduação após a conclusão da $\mathrm{RM}^{21}$. Atualmente, a produção de pelo menos um estudo apresentado em um congresso da especialidade passou a ser condição obrigatória para conclusão do programa.

Em 2018, seis em cada dez médicos possuíam títulos de especialista no Brasil, apontando que os médicos brasileiros estão cada vez mais cientes da importância da especialização 
para conquistar boas oportunidades de carreira ${ }^{10}$. No nosso estudo, destacamos que $75,94 \%$ dos egressos das áreas básicas cursaram ou estavam cursando um segundo PRM, achado também presente no trabalho de Pinto et al. ${ }^{17}$, que mostrou que $80,7 \%$ dos egressos de cirurgia geral também cursaram uma segunda RM.

Tradicionalmente, a residência é o lócus privilegiado da formação de pós-graduação em Medicina. Entretanto, nos últimos anos, o prestígio alcançado pela pós-graduação stricto sensu vem aumentando o interesse dos profissionais médicos ${ }^{22}$. Na nossa pesquisa, $24,06 \%$ dos egressos já eram portadores do título de mestre. Vale ressaltar que esse resultado deve ter sido influenciado pelo fato de o IMIP ser uma das poucas instituições do Brasil a oferecer o programa de mestrado associado à residência.

Morrison et al. ${ }^{23}$ em 2001 descreveram que 55\% dos PRMs dos Estados Unidos incluíam no seu currículo o treinamento de habilidades docentes, com os programas resident-as-teacher, que ganharam relevância desde a década de 1990. No Brasil, não existe programa similar, entretanto, na nossa pesquisa, 26,58\% dos egressos também já atuavam como docentes em uma IES de graduação em Medicina.

A análise do perfil profissional verificou que 93,7\% dos egressos exercem a especialidade da residência cursada principalmente no estado de Pernambuco (68,4\%). A maior parte exerce suas atividades tanto na rede pública quanto na rede privada, o que parece ser a maneira de atuação mais comum do médico jovem. Ressaltamos que 76 egressos (96,20\%) exercem a medicina em alguma instituição da rede pública, evidenciando que, independentemente da área de atuação, os PRMs têm contribuído efetivamente para a formação de profissionais capacitados para o SUS ${ }^{24}$.

Quanto à quantidade de horas de trabalho semanais, cerca de $43 \%$ trabalham entre 40 e 60 horas. Esse dado apresenta grande variação entre diversos estudos. A análise dos médicos recém-formados no Sul do Brasil demostrou que eles trabalhavam majoritariamente até 40 horas por semana ${ }^{25}$, enquanto um estudo sobre o perfil de trabalho dos médicos de um programa de residência tradicional chinês verificou uma média de 83,28 horas semanais entre os egressos da Peking Union Medical College Hospital, em Pequim, na China ${ }^{26}$.

A renda mensal atual derivada do trabalho médico recebida por mais da metade dos egressos (55,69\%) encontrase na faixa de dez a 20 salários mínimos nacionais. Esses dados são superiores à média salarial mensal dos médicos no âmbito nacional, em $2018^{10}$.

Verificamos aspectos relacionados à satisfação do PRM cursado. Cerca de $75 \%$ dos egressos afirmaram que cursariam o programa novamente na instituição e declararam que a realização da residência no IMIP facilitou a vida profissional deles. Esses dados são indicativos da consolidação da qualidade do ensino oferecido no serviço ${ }^{27}$. Entre os aspectos positivos identificados nos PRMs, foram relevantes o número de pacientes, a diversidade de casos e o bom envolvimento dos preceptores. Os preceptores desempenham papel vital na preparação dos residentes, pois são eles que transmitem experiência e confiança e procuram fornecer oportunidades para o desenvolvimento das competências dos alunos em treinamento ${ }^{20}$. A principal desvantagem elencada foi a carga horária excessiva, relatada por $11,39 \%$ dos egressos.

Avaliou-se a percepção desse jovem médico sobre sua qualidade de vida. A maioria dos participantes respondeu que a profissão atendeu às expectativas geradas durante o curso e considera gratificante o exercício da profissão. No entanto, $59,49 \%$ dos médicos contestaram a afirmação de que o seu tempo livre é suficiente para cuidar da saúde física e mental, e $50,63 \%$ relataram não praticar exercícios físicos, fator que pode estar relacionado ao aumento de peso relatado por $33(41,77 \%)$ dos egressos após o término da residência ${ }^{28}$.

\section{LIMITAÇÕES DO ESTUDO}

Embora os questionários eletrônicos sejam uma ferramenta prática e factível para obter informações dos egressos de programa de residência, o percentual de respostas é sempre um fator limitante dessa metodologia. Além disso, o fato de ser um estudo realizado em um único centro pode não permitir extrapolações para egressos de outros locais.

\section{CONCLUSÕES}

A monitoração periódica de egressos de programa de residência é um instrumento útil para a avaliação do programa e permite o monitoramento das intervenções implementadas, viabilizando inclusive a obtenção de informações que ajudem no planejamento de novos programas. O estudo confirmou que a RM contribui para a fixação do profissional na região do programa cursado e a grande maioria dos egressos exerce a sua especialidade. As atividades laborais são exercidas com frequência no SUS, tendo a residência contribuído de forma efetiva para a qualificação da assistência.

O conteúdo de atividades de ensino de especialidades necessita de constante revisão, e os resultados do nosso estudo foram essenciais para a discussão de mecanismos internos de avaliação e de indicadores indiretos da qualidade da formação dos PRM da instituição.

\section{CONTRIBUIÇÃO DOS AUTORES}

Eduardo Jorge da Fonseca Lima participou da concepção e do desenho deste estudo, da análise e interpretação dos dados, e da redação e revisão da versão final deste artigo. Pedro Jorge 
Serra da Fonseca Lima, Pedro Henrique Alves de Andrade e Lucas Miranda Castro participaram da concepção deste estudo, da coleta e análise dos dados, e da revisão da versão final deste artigo. Afra Suassuna Fernandes participou da concepção deste estudo e da revisão da versão final deste artigo.

\section{CONFLITO DE INTERESSES}

Declaramos não haver conflito de interesses neste estudo.

\section{FINANCIAMENTO}

Declaramos que não houve financiamento neste estudo.

\section{REFERÊNCIAS}

1. Brasil. Residência médica. Brasília: Ministério da Educação; c2018 [acesso em 28 abr 2018]. Disponível em: http://portal.mec.gov.br/residencias-emsaude/residencia-medica.

2. Magalhães APS, Esteves CC, Elias SF, Oliveira LD, Figueredo NDM, Costa ID. Perfil dos egressos de medicina de uma Faculdade de Medicina de Juiz de Fora/MG. Rev Ciênc Saúde. 2012;2(2):32-44.

3. Belmar C. Fernando Figueira: o homem que arrastou rochedos. São Paulo: Escrituras; 2007.

4. Maniglia JV. Perfil do egresso da residência em otorrinolaringologia e cirurgia de cabeça e pescoço da Santa Casa de Franca, da Faculdade de Medicina de São José do Rio Preto e da Clínica Maniglia. Arq Ciênc Saúde. 2004;11(1):29-36.

5. Freed GL, Dunham KM, Switalski KE, Jones Jr MD, McGuinness GA. Research Advisory Committee of the American Board of Pediatrics. Recently trained general pediatricians: perspectives on residency training and scope of practice. Pediatrics. 2009;123(Suppl 1):S38-S43.

6. Focaccia R, Elias PM, Amato Neto V. Residência médica em doenças infecciosas e parasitárias no Hospital das Clínicas da Faculdade de Medicina da Universidade de São Paulo. Rev Hosp Clin Fac Med Univ São Paulo.1988;43:171-5.

7. Lampert JB. Educação médica no século XXI: mudanças no perfil do egresso. Rev Bras Educ Med. 2014;38(3):291-2.

8. Conselho Regional de Medicina do Estado de São Paulo. Estado financia residência, mas regula pouco a oferta de profissionais. Informativos do CREMESP. 2008;255:1-21 [acesso em 19 set 2020]. Disponível em: http:// www.cremesp.org.br/library/modulos/centro_de_dados/arquivos/ residencia_medica.pdf.

9. Koch VHK, Doria Filho U, Bollela VR. Avaliação do programa de Residência Médica do Departamento de Pediatria da Faculdade de Medicina da Universidade de São Paulo. Rev Bras Educ Med. 2011;35(4):454-9 [acesso em 19 set 2020]. Disponível em: https://www.scielo.br/scielo. php?script=sci_abstract \&pid=S0100-55022011000400003\&lng=en\&nrm =iso\&tlng=pt.

10. Scheffer M, coordenador. Demografia médica no Brasil 2018. São Paulo: FMUSP; 2018.

11. Lima FD, Buunk BP, Araújo BMJ, Chaves JGM, Muniz DLO, Queiroz LB. Síndrome de burnout em residentes da Universidade Federal de Uberlândia. Rev Bras Educ Med. 2007;31(2):137-46.
12. Observatório de Recursos Humanos em Saúde de São Paulo. A inserção dos egressos dos programas de residência médica financiados pelo governo do Estado de São Paulo no mercado de trabalho. São Paulo: ObservaRHSP [acesso em 28 abr 2018]. Disponível em: http://www. observarh.org.br/observarh/repertorio/Repertorio_ObservaRH/SES-SP/ Egressos_residencia_medica.pdf.

13. Brasil. Programas de residência têm novas diretrizes publicadas em resolução. Brasília: Ministério da Educação; 2012 [acesso em 30 jul 2019]. Disponível em: http://portal.mec.gov.br/ultimas-noticias/212-educacaosuperior-1690610854/17683-programas-de-residencia-tem-novasdiretrizes-publicadas-em-resolucao.

14. Smith KL, Tichenor CJ, Schroeder M, Jensen GM. Orthopaedic residency training: a survey of the graduates $\bigotimes$ perspective. J Orthop Sports Phys Ther. 1999;29(11):635-51.

15. Scheffer MC, Jones A, Cassenote F. A feminização da medicina no Brasil. Rev Bioét. 2013;21(2):268-77.

16. Machado MDC. A feminização da medicina. Anal Soc. 2003;38(166):127-37.

17. Pinto FCF, Ferreira JBB, Caritá EC, da Silva SS. Perfil dos egressos da residência médica em cirurgia geral de uma universidade do interior paulista. Rev Bras Educ Med. 2018;42(4):144-54.

18. Nunes MPT, Michel JLM, Brenelli SL, Haddad AE, Mafra D, Ribeiro ECO, et al. Distribuição das vagas de residência médica e de médicos nas regiões do país. Cad Abem. 2011;7:28-34.

19. Petta HL. Formação de médicos especialistas no SUS: descrição e análise da implementação do programa nacional de apoio à formação de médicos especialistas em áreas estratégicas (Pró-Residência). Rev Bras Educ Med. 2013;37(1):72-9.

20. Batista KT, Pacheco LMS, Silva LM. Avaliação dos programas de residência médica em cirurgia plástica no Distrito Federal. Rev Bras Cir Plást. $2011 ; 28(1): 20-8$.

21. Grinberg $M$, Nunes Filho ACB. Iniciação científica, residência médica e investigação clínica. Arq Bras Cardiol. 2011;97(1):e11-e12.

22. Coser O. Expectativas de aprimoramento pós-residência médica: hora para um mestrado profissional? Rev Bras Educ Med. 2012;36(93):325-34.

23. Morrison EH, Friedland JA, Boker J, Rucker L, Hollingshead J, Murata P. Residents-as-teachers training in U. S. residency programs and offices of graduate medical education. Acad Med. 2001;76(10 Suppl):S1-S4.

24. Brasil CC, Oliveira PRS, Morais APSV. Perfil e trajetória profissional dos egressos de residência multiprofissional: trabalho e formação em saúde. Sanare. 2017;16(1):60-6.

25. Purim KSM, Borges LMC, Possebom AC. Perfil do médico recém-formado no Sul do Brasil e sua inserção profissional. Rev Col Bras Cir. 2016;43(4):295-300.

26. Zhang $Y$, Huang $X$, Li H, Zeng $X$, Shen T. Survey results of job status of residents in a standardized residency training program. BMC Med Educ. 2019;19(1):281-291.

27. Instituto de Medicina Integral Professor Fernando Figueira. Diretoria de ensino. Recife: IMIP; 2015 [acesso em 31 jul 2019]. Disponível em: http://www. informazione4.com.br/cms/opencms/imip/pt/ens_pesq_ext/ensino/.

28. Mota MC, De-Souza DA, de Mello MT, Tufik S, Crispim CA. Estilo de vida e formação médica: impacto sobre o perfil nutricional. Rev Bras Educ Med. 2012;36(3):358-68. 